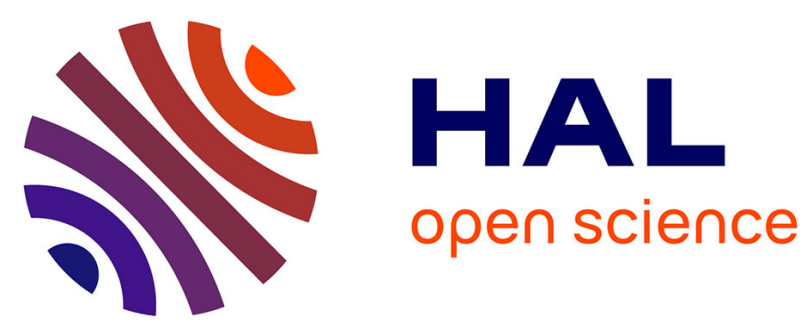

\title{
Mobility Beamforming Prediction and a Round Robin Scheduling in a Directional MAC for MANET
}

Vincenzo Inzillo, Floriano de Rango, Alfonso Ariza Quintana

\section{To cite this version:}

Vincenzo Inzillo, Floriano de Rango, Alfonso Ariza Quintana. Mobility Beamforming Prediction and a Round Robin Scheduling in a Directional MAC for MANET. 11th IFIP Wireless and Mobile Networking Conference (WMNC 2018), Sep 2018, Prague, Czech Republic. pp.25-31. hal-01995162

\section{HAL Id: hal-01995162 \\ https://hal.inria.fr/hal-01995162}

Submitted on 25 Jan 2019

HAL is a multi-disciplinary open access archive for the deposit and dissemination of scientific research documents, whether they are published or not. The documents may come from teaching and research institutions in France or abroad, or from public or private research centers.
L'archive ouverte pluridisciplinaire HAL, est destinée au dépôt et à la diffusion de documents scientifiques de niveau recherche, publiés ou non, émanant des établissements d'enseignement et de recherche français ou étrangers, des laboratoires publics ou privés. 


\section{Mobility Beamforming Prediction and a Round Robin Scheduling in a Directional MAC for MANET}

\author{
Vincenzo Inzillo \\ DIMES, University of Calabria \\ Via P.Bucci, Rende (CS), Italy \\ Email:v.inzillo@dimes.unical.it
}

\author{
Floriano De Rango \\ DIMES, University of Calabria \\ Via P.Bucci, Rende (CS), Italy \\ Email: derango@dimes.unical.it
}

\author{
Alfonso Ariza Quintana \\ University of Malaga \\ Av. De Cervantes, Malaga, Spain \\ Email: aarizaq@uma.es
}

\begin{abstract}
In pervasive directional beamforming mobile network environments, the mobility of nodes could bring to an excessive loss of synchronization between couples of nodes which are performing a communication; this issue is also known as handoff problem. This kind of problem usually contribute to dramatically reduce the network performance especially at MAC (Medium Access Control) layer. Although sectorized approaches could be used in order to limit this issue, an high accuracy in the estimation of the future position of the nodes is required for granting the mitigation of the handoff and at the same time an overall improvement of the network performance. In view of this, we propose a versatile predictive location mechanism that provides for an high-efficiency tracking of the position of the nodes by using a frame scheduler with priority.
\end{abstract}

Index Terms-Beamforming, MANET, Mobility, Handoff

\section{INTRODUCTION}

Mobile Ad hoc Networks (MANET) are self-organized networks in which mobile nodes can move independently; the nodes usually move according to a certain mobility pattern model and the movement of a node is not necessarily related to the movement of other nodes in the network. The employment of directional antennas and the development of strategies that allow to achieve an high degree of scalability with increasing of the number of active connections as well as the introduction of Smart Antenna Systems (SAS) in place of the classical omnidirectional approach addressed a lot of different challenges in the design of an efficient Medium Access Control (MAC) protocol; although the use of a directional approach, allows the achievement of large amount of energy saving in the network, it could introduce some issues that need to be deeply investigated, such as the hidden terminal problem and the deafness problem. The deafness problem [1] is a very common issue in which a particular node (the deaf node) is unaware of the signals from some other node, while it beamformed in another direction resulting in multiple packet drops due to increase in the back off time to a limit. Prolonged deafness can cause multiple packet drops, and also affects the fairness among transmitting nodes. Chains or Deadlock is possible when no nodes can communicate because of deafness. This issue could become very heightened as the mobility of the nodes increases. In particular, the performance of MAC protocols commonly degrades with increasing system dynamics, and malfunctions may occur in highly dynamic

ISBN 978-3-903176-03-4 2018 IFIP scenarios. When a node is subjected to mobility during a communication, especially in beamforming network contexts, it is highly likely that it can be affected by the handoff issue [2]. The problem of handoff, arises when a receiver node moves out of the beam of the transmitter. Moreover, in wireless systems, the channel can vary rapidly over time.

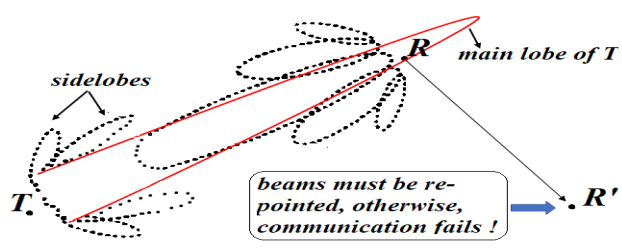

Fig. 1: Handoff in beamforming networks.

Fig. 1 illustrates an example of handoff: the transmitter node $\mathrm{T}$ is communicating with a node $\mathrm{R}$; during the communication $\mathrm{R}$ moves in the position $\mathrm{R}^{\prime}$ and exit out of the transmitter beam and consequently the communication fails and the beams need to be re-pointed. In this case, if the node in the position $\mathrm{R}^{\prime}$ can still be reached by $\mathrm{T}$ through a beam switching, we can refer to this operation as inter-beam handover. A most common solution used to mitigate the impact of the nodes mobility in directional communications is mobility prediction. Essentially, the mobility prediction is a method for estimating the trajectory of the future position of the nodes. Mobility prediction has been studied in cellular networks and wireless ad-hoc networks and represents a precious instrument for estimating the future location, mobility speed, and the direction of the mobile users [3]. Several analytical formulations have been proposed for modelling the mobility prediction; for example, the most common methods and approaches are based on the Markov model [4] and Hidden Markov Models (HMM) [5]. However they are not generic enough in order to face all types of mobility. In this paper, we propose a model for limiting the handoff issue together with the deafness problem in mediumhigh mobility beamforming wireless network environments in which directional sectorized MAC protocols are used. The relevance and the topicality of these issues related to the next generation MAC protocols design have motivated the purpose of this work. The paper is structured as follows: section II is an overview about the most important related works regarding deafness and handoff issues. In section III, the proposed model is presented. In section IV simulation results are discussed. 
Finally, section V summarizes the conclusions obtained by the experimental analysis.

\section{RELATED WORKS}

The deafness and the handoff issues were deeply investigated and treated in the latest years and, especially thanks to the growth of employment of the Smart Antenna Technology, a remarkable number of researches have been encouraged to propose novel solutions for minimizing or, at least, reducing these problems. In [6] a novel Deafness-Aware (DAMAC) is presented, in order to mitigate the deafness problem through a discrete-time Markov chain model. The model uses a double-channel schema in order to handle the Directional RTS (DRTS). In [7] authors, propose a New Deafness Aware (NDA-MAC) mechanism which tries to mitigate the deafness problem through the use of control channels and logical data; more specifically, a discrete time Markov chain model for evaluating the deafness impact is provided. A packet delivery scheduling algorithm and two MAC protocols in which nodes use directional sectorized antennas are proposed in [8]. In work [9] a Deafness-Free MAC protocol is presented; the deafness is encountered using localization information table for each node and the Code Division Multiple Access (CDMA) technique. However, these works address only the deafness problem without considering the effects of the mobility. Other approaches, such as the work [10], attempt to reduce the handoff issue through an efficient beam system control; a similar work is presented in [11]; in this paper, authors try to mitigate the handoff problem by proposing a predictive location model in order to advance the future position movements of the nodes. Nevertheless, these works are suitable for Vehicular ad Hoc Network (VANET) environments and do not properly address the deafness problem which is very relevant in MANET. For this reason, we propose an approach that could be useful for limiting either the deafness and either the handoff problem in beamforming contexts which provide for directional MAC communications.

\section{PROPOSED MODEL}

We propose to extend the Round Robin Medium Access Control (RR-MAC) approach presented in [12] in order to manage a situation which involves the movement of such node in the network.

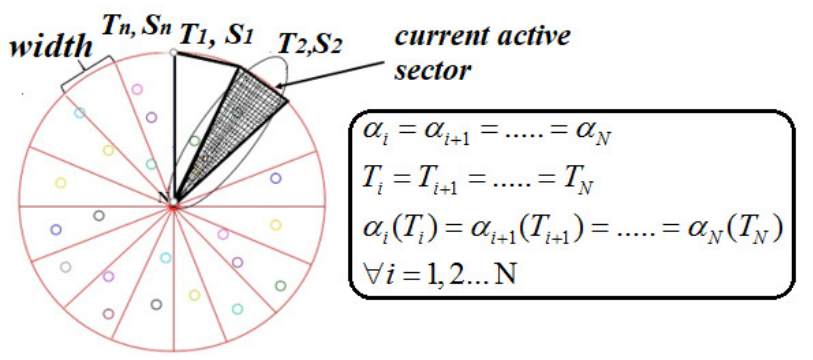

Fig. 2: Plane sectorization principle.

Fig. 2 illustrates the principle of the RR-MAC. RR-MAC provides for a sectorization of the plane into $N$ equal sectors (each sector is denoted by $S$ ), each one having a certain amplitude $w$ (sector width) and a beamforming duration time $T$ (sector time). Note that all sectors have the same width as well as the same sector time. Each node that belongs to a certain sector beamforms with an angle $\alpha_{i}$ where $i=1 \ldots N$ and $N$ is the number of sectors in which the plane is divided; each node beamforms in a certain sector until the sector time is reached, then it switches to the next sector. The pseudo-code of the algorithm is displayed as follows.

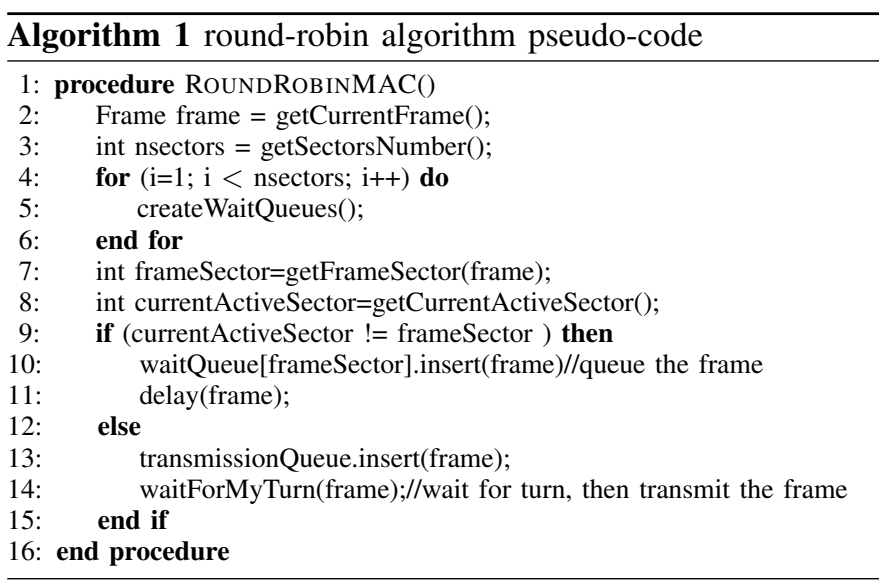

Each frame is processed by the system that checks if the sector for which is destined the frame is the current active sector. If true, it inserts the frame in the transmission queue and transmits the frame according to Last In First Out (LIFO) queue mechanism; if the direction of the receiver for which the current frame is destined is outside the set of the directions currently available (current active sector), the frame is queued in its waiting sector queue, and delayed until its sector does not become the current active sector. As it can be deduced, this model is perfectly suitable for limiting the deafness problem in beamforming networks, but does not consider the effects introduced by the mobility of nodes. For this reason we choose to extend this approach in order to adapt it also for mediumhigh mobility environments.

\section{A. Predictive Location Model}

For estimating the next position of a mobile node we basically use the model proposed in [11] that provides for a predictive location algorithm named TRAC (Triangle and Circle).

Fig. 3 synthesizes the operational principle of TRAC. Basically, each node stores in a history cache its current position together with the location of the previous position occupied. The algorithm provides for the estimation of the next position in which a node will move based on history cache info. Let us consider a certain transmitter node $T$ that is sending data towards a receiver node $R$, with $\mathrm{R}$ that moves according to a mobility pattern (in our case we used the Mass mobility model provided by Omnet++ simulator [17]). The current position of the node $R$ is denoted by $B$ and its location is represented by the coordinates $\left(x_{i}, y_{i}\right)$ while the previous location of $R$ is denoted by $A$ and has coordinates $\left(x_{i-1}, y_{i-1}\right)$; the distance 


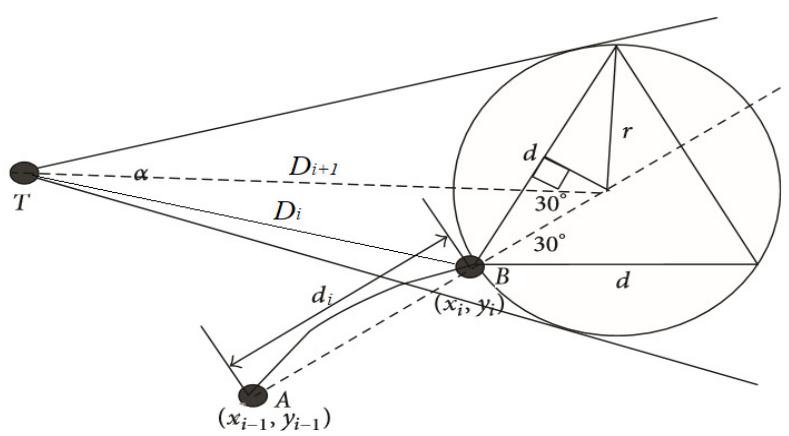

Fig. 3: TRAC operational principle.

between the transmitter and the i-esimum position of the receiver is denoted by $D_{i}$ while $d_{i}$ is the distance between $A$ and $B$. TRAC algorithm estimates the next position of node $R$ based on these location infos; in particular, the next position is approximatively within a circle that contains an equilateral triangle having one of its vertex coinciding with the position $B$. This search area is estimated by using the following steps:

1) calculate the slope of $\overrightarrow{A B}$ denoted by $k$ :

$$
k=\frac{y_{i}-y_{i-1}}{x_{i}-x_{i-1}}, \quad x_{i} \neq x_{i-1}
$$

2) make an equilateral triangle whose one vertex is at $B$ position centered at coordinates $\left(x_{i+1}, y_{i+1}\right)$ and side length $d$ expressed by:

$$
\begin{aligned}
& \left\{\begin{array}{l}
x_{i+1}=x_{i}+\frac{\sqrt{3} d}{3} \frac{x_{i}-x_{i-1}}{\left|x_{i}-x_{i-1}\right|} \cos (\arctan (|k|) \\
y_{i+1}=y_{i}+\frac{\sqrt{3} d}{6} \frac{y_{i}-y_{i-1}}{\left|y_{i}-y_{i-1}\right|} \cos (\arctan (|k|)
\end{array}\right. \\
& d=\sqrt{\left(x_{i}-x_{i-1}\right)^{2}+\left(y_{i}-y_{i-1}\right)^{2}}
\end{aligned}
$$

3) draw a circle that contains the equilateral triangle with radius:

$$
r=\frac{\sqrt{3} d}{3}
$$

4) calculate the needed sector angle as:

$$
\alpha=2 * \arcsin \left(\frac{r}{D_{i+1}}\right)
$$

Where $D_{i+1}$ is the approximate distance from the transmitter $T$ to the new position of the receiver node $R$.

\section{B. Enhanced TRAC algorithm}

Although TRAC algorithm results very functional for adaptive beamforming systems it presents some aspects that need to be investigated in the analysis of directional communications concerning dynamic environments such as MANET. Primarily, the TRAC algorithm does not consider the effects of the mobility speed of nodes that could affect the estimation of the next position of the node R; furthermore, it does not find any radio propagation channel model which could offer meaningful indications for improving the predictive position estimation.

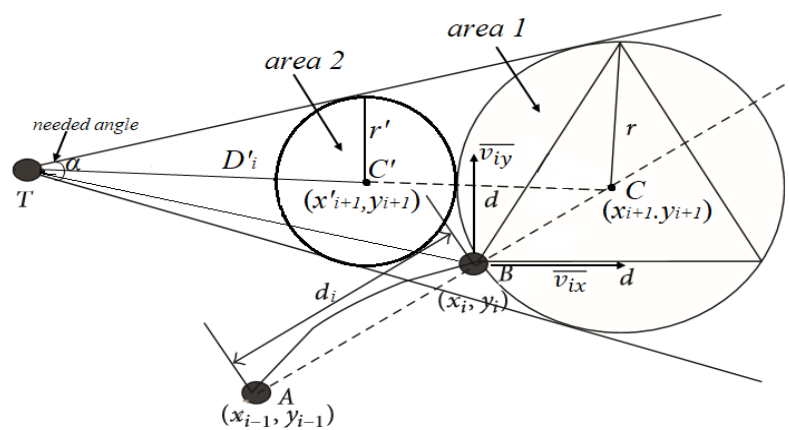

Fig. 4: Enhanced TRAC principle.

For this reason, we propose to enhance the TRAC algorithm by attempting to address these issues.

Fig. 4 illustrates the Enhanced TRAC principle. The new model now considers the mobility speed vector related to the current position $\bar{v}$ that consists of two vectorial components along $x$ and $y$ axes which could be expressed as:

$$
\left\{\begin{array}{l}
\overline{v_{i x}}=\frac{x_{i}-x_{i-1}}{\Delta t} \\
\overline{v_{i y}}=\frac{y_{i}-y_{i-1}}{\Delta t}
\end{array}\right.
$$

Where $\Delta t$ is the period time between two consecutive movements of the node $R$. Considering the dependence on the mobility speed vector the eq. 2 becomes:

$$
\left\{\begin{array}{l}
x_{i+1}=x_{i}+\frac{\sqrt{3} d}{3} \frac{\overline{v_{i x}} \Delta t}{\left|x_{i}-x_{i-1}\right|} \cos (\arctan (|k|) \\
y_{i+1}=y_{i}+\frac{\sqrt{3} d}{6} \frac{\overline{v_{i y}} \Delta t}{\left|y_{i}-y_{i-1}\right|} \cos (\arctan (|k|)
\end{array}\right.
$$

As it can be noted, the coordinates of the next position of the node are now function of the mobility speed. In order to improve the accuracy in the position estimation we can consider the Friis radio propagation model:

$$
P_{R X}=P_{T X}\left(\frac{\lambda}{4 \pi d L}\right)^{2}
$$

Where $P_{R X}$ is the power related to the receiver, $P_{T X}$ is the transmitted power, $\lambda$ is wavelength, $d$ is the distance between the transmitter and the receiver while $L$ denotes the losses in the channel. Based on this equation we can evaluate the approximate distance between the transmitter node $T$ and the receiver $\mathrm{R}$ in the new position. More specifically, let us assume to check the received power at node $R$ every time that it changes its current position; considering the eq. 8 , the distance between the transmitter node $T$ and the receiver $R$ it depends on the received power; In particular, the search area of the new position of node $R$ depends on the received power evaluated in its new position. In Fig. 4 we can distinguish two main search areas: the first one that we named area $l$ having center at position $C$ and another one named area 2 centered at position $C^{\prime}$ having radius $r^{\prime}=\sqrt{3} d / 6$ and coordinate component along $\mathrm{x}$ axis that can be expressed as:

$$
x_{i+1}^{\prime}=\left(x_{i}-d\right)+\frac{\sqrt{3} d}{3} \frac{\overline{v_{i x}} \Delta t}{\left|x_{i}-x_{i-1}\right|} \cos (\arctan |k|)
$$


The distance between the two centers $C$ and $C$ ' is approximatively $d$. The new position of node $R$ depends on the received power measured at this position. Let $P$ be the new position of the node $\mathrm{R}$ and $P_{R X}(P)$ the received power of the node $R$ in the position $P$, we can locate the approximate search area of the position $P$ as follows:

$$
\begin{cases}P_{R X}(P) \leq P_{R X}(B)+\Delta(L) & P \in \text { area } 1 \\ P_{R X}(P)>P_{R X}(B)+\Delta(L) & P \in \text { area } 2\end{cases}
$$

Where $P_{R X}(B)$ is the received power of the node $R$ at the position $B$ while $\Delta(L)$ is a term that is function of the losses in the channel. Because of the use of the Friis model it is important to notice that the expression 10 is theoretically suitable for Line of Sight (LoS) scenarios having $\Delta(L)=0$. Therefore, the real environments are typically referred to nonLoS situations, therefore the accuracy of the nodes position estimation is certainly related to the number of losses in the scenario. In order to overcome this kind of issue, for directional communications it is possible to estimate the attenuation in function of the main beam orientation [14], [15]. For this purpose, we created the attenuation database in which the position and direction infos related to an incoming communication are cached and periodically updated; these informations are used to improve the accuracy of the estimation. From different samples of simulations we derived that the interval of values that maximizes the accuracy of the estimation is reached for $0.12 P_{R X}(B) \leq \Delta(L) \leq 0.16 P_{R X}(B)$. By these expedients, the estimation of the new position of the node $R$ can result more accurate with respect to the original TRAC formulation.

\section{Enhanced RR algorithm based on mobility-aware priority}

Once designed the predictive location model we can use it in order to improve our Round-Robin algorithm, providing a mechanism for mobility network management. From work [12] it can be noted that the performances of the system in terms of fairness are very related to the sector width value.

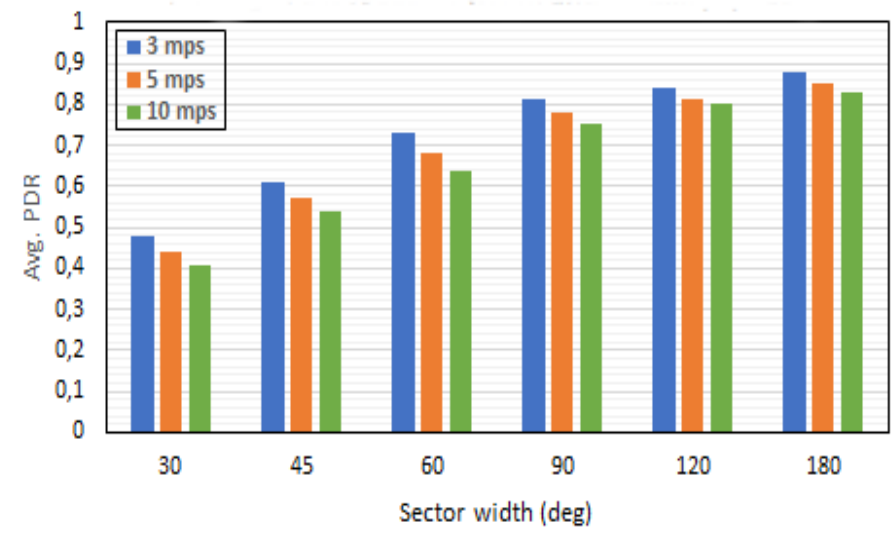

Fig. 5: Average PDR vs mobility speed RR-MAC.
In Fig. 5 it can be highlighted that, as the sector width decreases, the average Packet Delivery Ratio (PDR) also decreases. In particular, the RR algorithm provides for a good PDR value in slow mobility speed conditions but as the speed increases it tends to dramatically decreases for sector width values less than $60^{\circ}$. In view of this, we design a mechanism that extends the Round-Robin algorithm in order to minimize this situation. The next figure illustrates the Enhanced RoundRobin principle:

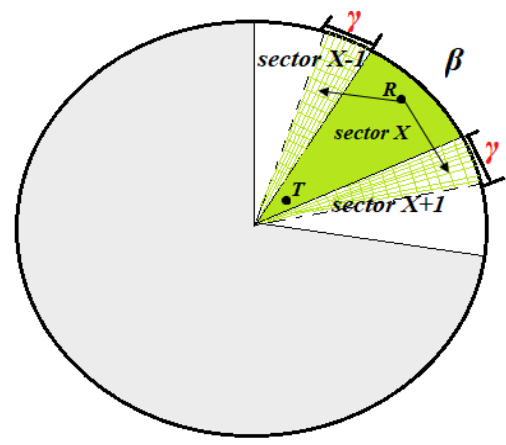

Fig. 6: Enhanced Round-Robin algorithm principle.

Fig. 6 shows an example in which the sector $x$ is the current active sector and each sector presents a sector width value $\beta=30^{\circ}$. Let us assume that the node $T$ is transmitting towards the node $R$ and that node $R$ moves in the network changing its sector. It will move in the sector $x-1$ or in the sector $x+1$ with same probability. The next position in which the node $R$ will move is estimated by the Enhanced TRAC algorithm that also estimates the needed angle $\alpha$; the Enhanced RR algorithm provides for a temporary enlargement of the current sector beam if the node $T$ senses a variation of the received power of $R$ during the communication, that could be interpreted as a change of position of node $R$. This enlargement is produced for a time $T_{e n}$ that is a fraction of the sector time $T_{s}$; in particular, based on results obtained after a set of simulations, we considered $T_{e n}=0.25 T_{s}$; the enlargement angle $\gamma$ is evaluated as:

$$
\gamma= \begin{cases}\left|\frac{\alpha-\beta}{2}\right| & \alpha>\beta \\ \beta & \alpha \leq \beta\end{cases}
$$

From eq. 11 it can be seen that, the enlargement angle depends on the needed angle $\alpha$ that is evaluated by the Enhanced TRAC algorithm; if this value is less or equal to the sector width $\beta$, no enlargement is provided. The Enhanced Round-Robin algorithm also addresses the handoff problem. In order to face up to this issue we use a priority scheduling that assigns a certain priority level to each kind of communication. These levels vary from 1 to 3 , where 1 represents the highest priority level. More specifically, the priority values are assigned as follows:

- priority level 1: communications not completed in which either the transmitter or either the receiver move in a sector adjacent to the current active sector. 
- priority level 2: communications of the sector queues.

- priority level 3: communications in the current active sector.

The highest priority is assigned to communications related to nodes that move in a neighbor sector. In this case the communication continues, after a re-pointing process of the beams, until it does not completely finish; note that the change of sector of a node does not affect the continuity of the communication; in this way the fail induced by handoff is avoided. If there are no nodes in the current active sector that change their sector during a communication, the scheduler selects the communications that are in the sector queue. Once the sector queue is empty, the scheduler selects the communication of the current active sector that are not in the sector queue (lowest priority level).

\section{Model implementation and Simulation Results}

As instrument for our model implementation we chose the Omnet++ Simulator; we used the adaptive Smart Antenna PhasedArray module designed in [16]. For enabling the Round-Robin approach we extended PhasedArray module adding several configuration variables (such as sectorTime and sectorWidth) and a set of utility functions.

\section{A. RR-MAC vs Mobility Speed Simulation Results}

The first set of simulations is accomplished executing the original RR-MAC protocol proposed in [12] without using any predictive model for estimating the future position of nodes. The system performances are analysed in terms of PDR, number of collisions at MAC layer and DRTS/DCTS (Directional Request to Send Directional Clear to Send) ratio; this last parameter was defined in [12] and it could result very useful for the estimation of the fairness of the system. These results are examined by increasing the average nodes mobility speed from $3 \mathrm{mps}$ (meter per seconds) to $10 \mathrm{mps}$ and the sector time (st) from 1 to 4 seconds. The following table summarizes the main parameters set used in our simulations:

TABLE I: Simulation parameters set.

\begin{tabular}{|c|c|}
\hline Antenna Model & SAS phasedArray \\
\hline Array Elements Spacing & $0.5 \lambda$ \\
\hline Number of Nodes & $10,30,50$ \\
\hline Network Standard & IEEE $802.11 \mathrm{n}$ \\
\hline Transmission Rate & 54 Mbps \\
\hline Traffic data type & UDP \\
\hline Message Length & 512 Byte \\
\hline Simulation Area Size & $500 \times 500 \mathrm{~m}$ \\
\hline Simulation Time & $300 \mathrm{~s}$ \\
\hline Mobility model & Mass Mobility \\
\hline Node mobility speed values & $3,5,10 \mathrm{mps}$ \\
\hline Sector Time values & $1,2,3,4 \mathrm{~s}$ \\
\hline Sector Width values & $30^{\circ}, 45^{\circ}, 60^{\circ}, 90^{\circ}, 120^{\circ}, 180^{\circ}$ \\
\hline
\end{tabular}

We assumed that mobile nodes are uniformly distributed in the network; note that the obtained values are evaluated by estimating confidence intervals by varying the simulation seed from 1 to 20. Therefore, for reason of spaces the next figures illustrate the results related to the configuration case having number of nodes set to 30 .

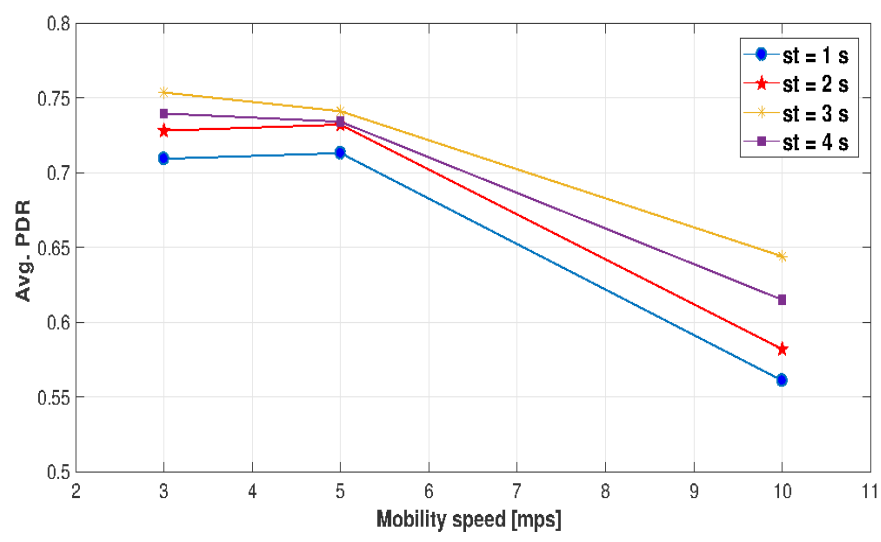

Fig. 7: Average PDR vs Mobility speed.

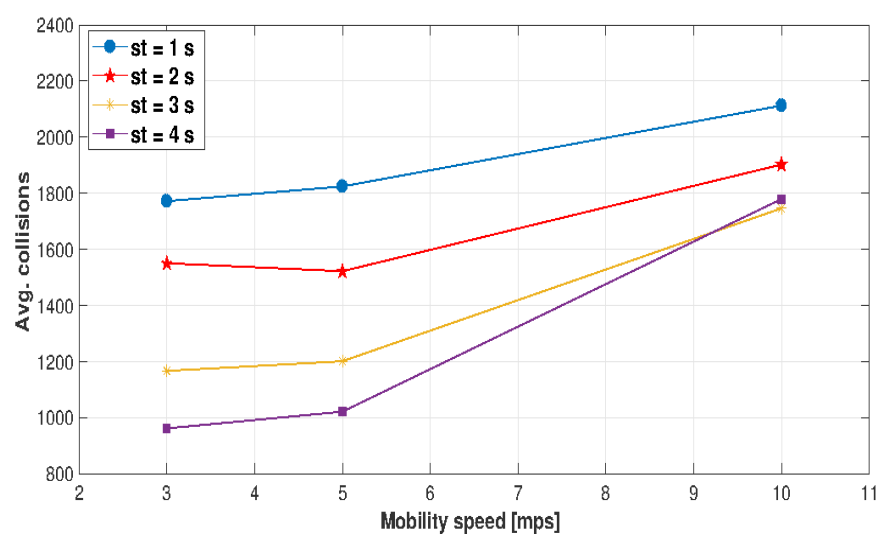

Fig. 8: Average Num. of Collisions vs Mobility speed.

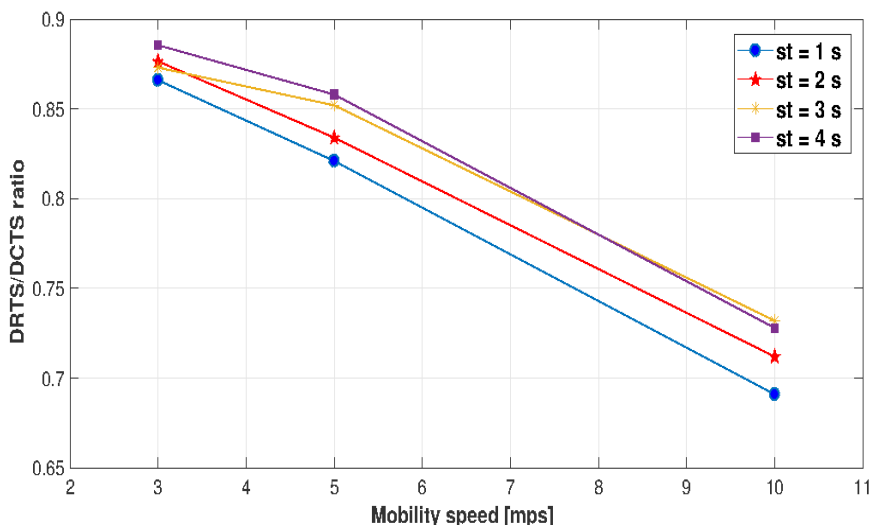

Fig. 9: DRTS/DCTS ratio vs Mobility speed.

Fig. 7 displays the statistic plot related to average PDR; the figure shows a slightly improvement of the PDR as the sector time increases; however as evidenced in [12] this trend is not linear and suffers from a decrease for sector values higher than $4 s$. From Fig. 7 it also can be noted that the PDR 
tends to reduce significantly as the mobility speed of nodes increases. This trend is confirmed from Fig. 8 in which it can be observed an increment of the number of collisions of $54 \%$ in the worst sector time case with respect the best case (st $=3 s$ ). In Fig. 9 it can be observed how the fairness of the system in terms of DRTS/DCTS ratio is quite good as long as the mobility speed is low; however this value decreases at an exponential rate for high speed values; in particular, in the mobility speed case related to $10 \mathrm{mps}$ it is registered a reduction of the DRTS/DCTS ratio of about $24 \%$ with respect to the lowest mobility speed considered case. In summary, the overall reduction of the system performance is probably due to the fact that, in this case, the system does not support any mechanism that handles the mobility of the nodes; in order to confirm this hypothesis the following table shows the average failed communications due to handoff in the network (in terms of percentage) obtained from simulations.

TABLE II: Failed communications due to handoff

\begin{tabular}{|c|c|c|}
\hline \multicolumn{3}{|c|}{ Failed handoff communications } \\
\hline $\mathbf{3}$ mps & $\mathbf{5}$ mps & $\mathbf{1 0}$ mps \\
\hline $11,42 \%$ & $18,01 \%$ & $34,6 \%$ \\
\hline
\end{tabular}

We considered as handoff communication a transmission/reception that presents at least a change of sector (by a transmitter or a receiver node). From Table II it can be deduced how the lack of a mobility management mechanism heavily influences the performance of the system; in particular, in the case of $10 \mathrm{mps}$ about one-third of the communications fail.

\section{B. Enhanced RR-MAC vs Mobility Speed Simulation Results}

As last analysis we enabled the modifications introduced to the original TRAC and RR algorithms. In this case, the scheduler uses either the designed predictive model (ETRAC) and either the priority mechanism provided by the Enhanced RR algorithm. The next figures illustrate the statistics related to PDR, number of collisions and DRTS/DCTS ratio.

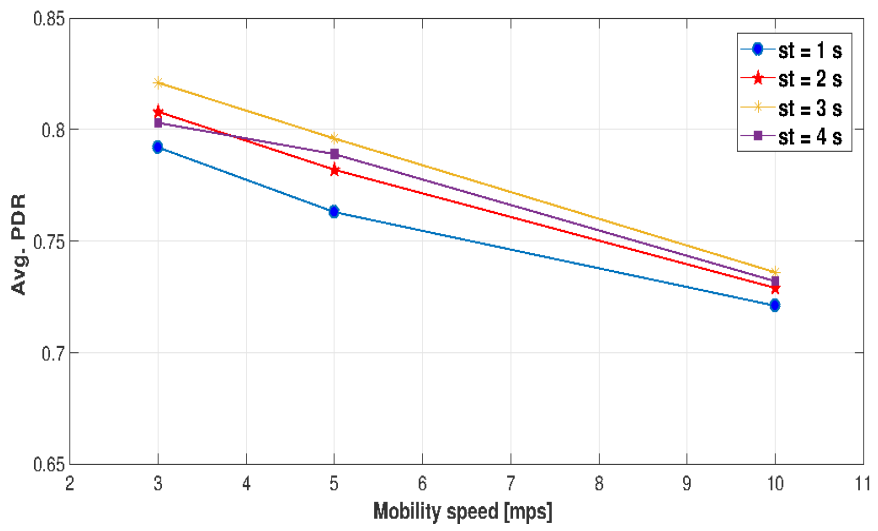

Fig. 10: Average PDR vs Mobility speed Enh. RR-MAC.

From Fig. 10 we observe that, the minimum value of PDR registered in the worst case is 0.721 against the 0.561 obtained

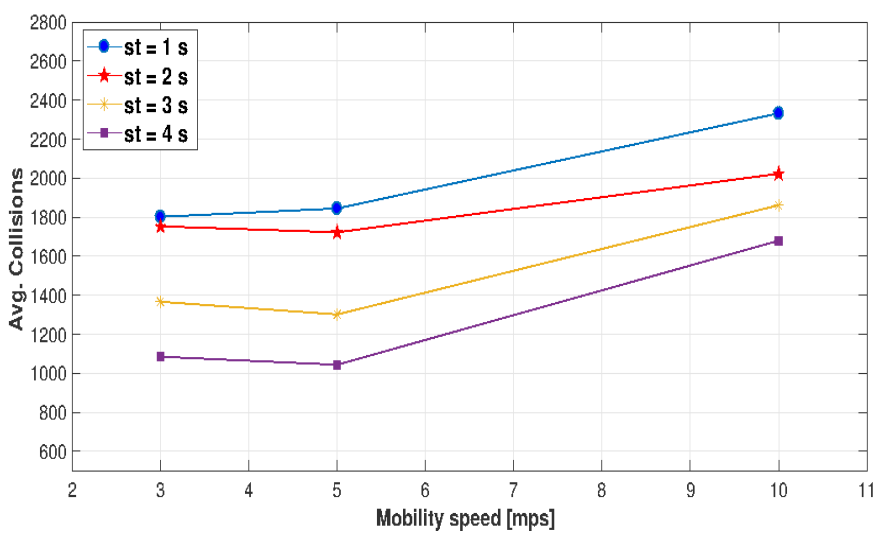

Fig. 11: Average Num. of Collisions vs Mobility speed Enh. RR-MAC.

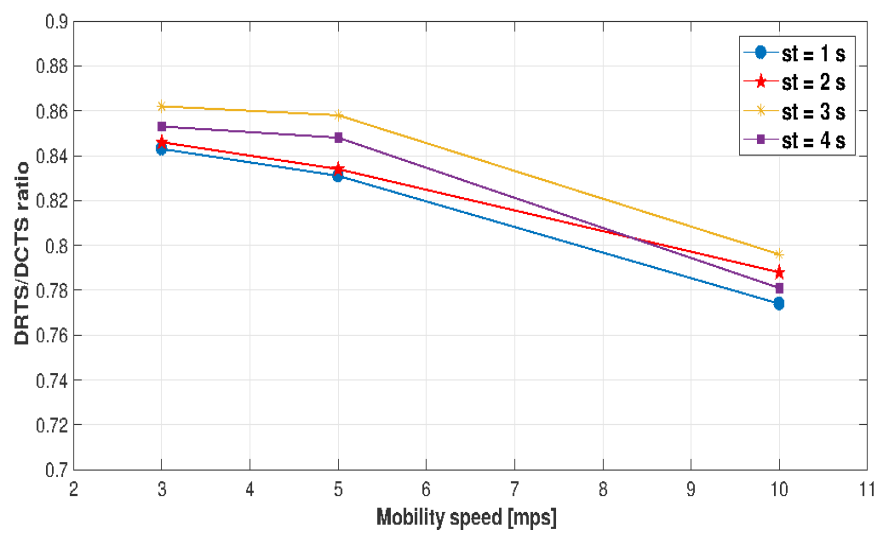

Fig. 12: DRTS/DCTS ratio vs Mobility speed Enh. RR-MAC.

in the original RR case. In Fig. 11 it may be experienced a little improvement of the average number of collisions for all of the considered cases; this behaviour is probably due to the beam enlargement involved by the Enhanced Round Robin algorithm which slightly extends the set of directions usually related to a certain sector; however, this improvement is about $2.5 \%$ with respect to the original RR-MAC and it results quite negligible. The Fig. 12 highlights that, in high mobility conditions (10 mps) it can be obtained a minimum DRTS/DCTS ratio of 0.774 against the 0.691 registered in the RR-MAC case; in particular, the average value by varying the mobility speed is about 0.826 , that improves considerably the value of 0.731 obtained in the RR case. This growth is certainly justified because of the priority mechanism provided by the scheduler that contributes to reduce the number of communications failed for handoff.

\section{Contribution of the proposed model and comparison with} related works

For evaluating the contribution of the present paper with respect to the current state of art, we perform a comparison between the proposed Enhanced Round Robin Algorithm features with respect to the most important related works cited in section II. For this purpose the following table collects some 
useful parameters or functions provided by all considered approaches:

TABLE III: Related works comparison scheme

\begin{tabular}{|c|c|c|c|c|}
\hline Work & deafness & handoff & mobility & scalability \\
\hline DA-DMAC & $\checkmark$ & $x$ & $x$ & $\checkmark$ \\
\hline NDA-MAC & $\checkmark$ & $x$ & $x$ & $x$ \\
\hline DF-MAC & $\checkmark$ & $x$ & $x$ & $\checkmark$ \\
\hline Soft Handoff Method & $x$ & $\checkmark$ & $\checkmark$ & $x$ \\
\hline RR-MAC & $\checkmark$ & $x$ & $x$ & $\checkmark$ \\
\hline Enh. RR-MAC & $\checkmark$ & $\checkmark$ & $\checkmark$ & $\checkmark$ \\
\hline
\end{tabular}

In Table III it can be seen that, protocols such as NDAMAC and DA-DMAC properly addresses the deafness issue while ensuring a substantial reduction of the overall MAC collisions. In addition, DA-DMAC well performs also for a large number of mobile nodes in the network as well as the DF-MAC approach. However, none of the above support a mechanism able to counteract the handoff problem that is certainly induced by the mobility of nodes in beamforming contexts. In spite of this, the Soft Handoff Method proposes a valid contribution in order to mitigate the handoff problem but unfortunately does not provide any feature for limiting deafness at MAC layer; therefore it is suitable for Vehicular Network environments. Again, our RR-MAC provides quite good results in terms of deafness reduction due that the high level of fairness achieved also with increasing the number of nodes; therefore, RR-MAC does not consider the effect of the mobility of the nodes in the network scenario and consequently does not address the handoff problem; this aspect is definitely improved by the Enhanced RR-MAC that is able to minimize the effect of the mobility speed of nodes through an efficient prediction position estimation method. Our contribution with respect to the existing models can be summarized by the following points:

1) We proposed a predictive position estimation model that enhances the TRAC algorithm presented in [11]. Our model provides for an high accuracy node position evaluation also for quite complex real scenarios that could be affected from a considerable number of losses related to the channel (non LoS environments).

2) We enhanced the sectorized MAC protocol designed in [12]. For this purpose, we designed an efficient algorithm that is able to avoid the excessive degradation of the network performance at MAC layer. The novel designed algorithm provides for a priority in-frame scheduling in order to achieve high requirements of Quality of Service $(\mathrm{QoS})$ for mobile nodes experiencing inter-beam handover.

3) We improved such features of the most recent related works in terms of MAC layer performance and scalability. In this regard we equipped the mobile nodes with an efficient SAS adaptive array hardware technology in spite of the classical directional antenna system used in the majority of the related works.
4) We enhanced the Omnet++ network simulator features by extending the directional sectorized MAC module functionalities that was planned in [12] which may results largely useful in the analysis of wireless network beamforming environments.

\section{CONCLUSION}

We proposed an approach that could limit the excessive reduction of the performance in medium-high mobility wireless beamforming networks environments. We demonstrated that, by introducing some mechanisms that properly handle the mobility of nodes, it is possible to mitigate the effect of a very common phenomenon that could occur at MAC layer such as the handoff problem. Results have shown that, by increasing the accuracy in the node position estimation, it is possible to improve the system performance especially in terms of fairness and PDR. However, the employment of this approach could produce a very small increase of the number of the collisions at MAC layer, that result quite negligible in spite of the overall enhancement registered for PDR and DRTS/DCTS ratio. Nevertheless, we provided for an helpful support able to emulate the SAS technology which could become largely employed in the development of MAC protocols of next generation.

\section{REFERENCES}

[1] A. Akthar, S. Ergen, "Directional MAC protocol for IEEE 802.11 ad based wireless local area networks, " Ad Hoc Networks, 2018.

[2] H. Zhang, M.H. Fong et al., "Handoffs and handoff selection in a wireless access network," U.S. Patent, 2014.

[3] R. Suraj et al., "Mobility prediction in mobile ad hoc networks using a lightweight genetic algorithm," Wireless Networks, 2016.

[4] L. Ghouti, "Mobility prediction in mobile ad hoc networks using neural learning machines," Simulation Modelling Practice and Theory, 2016.

[5] L. Qiao, Y. Ansari et al., "Big data driven hidden Markov model based individual mobility prediction at points of interest," IEEE Transactions on Vehicular Technology, 2017.

[6] W. Na, et al. , "Deafness-Aware MAC protocol for directional antennas Ad Hoc Networks," Ad Hoc Networks, 2015.

[7] N. Kaushik, K. Choudary , "New Deafness-Aware Mac Protocol for Directional Antennas in WANET'S," IJCA 2015.

[8] A. Swaminathan, et al. , "The design of a channel-access protocol for a wireless ad hoc network with sectored directional antennas," Ad Hoc Networks, 2012.

[9] H. Su, S. Mo, "A Robust Deafness-Free MAC for Directional Antennas in Ad Hoc Networks," Wireless Personal Communications, 2017.

[10] M. de la Chapelle, A. Monk , "Soft handoff method and apparatus for mobile vehicles using directional antennas," U.S. Patent No. 9,306,657. Washington, DC: U.S. Patent and Trademark Office, 2016.

[11] L. Xiaofeng, P. Lio et al. , "A Location Prediction Algorithm for Mobile Communications Using Directional Antennas ," Int. Journal of Distributed Sensor Networks, 2013.

[12] V. Inzillo, F. De Rango, A. Ariza Quintana, "A Sectorized Directional MAC Proposal for Mitigating Deafness and Energy Consumption in Mobile Ad Hoc Networks," IEEE CCNC, 2018.

[13] Omnet++ Network Simulator Version 5.0.

[14] H. Ghayvat, S. Mukhopadhyay, X. Gui "Issues and mitigation of interference, attenuation and direction of arrival in IEEE 802.15. 4/ZigBee to wireless sensors and networks based smart building," Measurement, Elsevier, 2016.

[15] A. Salam, M. Vuran, "Smart underground antenna arrays: A soil moisture adaptive beamforming approach," INFOCOM 2017.

[16] V. Inzillo, F. De Rango, A. Ariza Quintana, "A new Variable Error Metric Adaptive Beamforming Algorithm for Smart Antenna Systems," IWCMC, 2017. 